\section{MammaPrint-Tests: für welche Patientinnen sinnvoll - und für welche nicht?}

\author{
Ein Expertengremium der American Society of Clinical Oncology (ASCO) \\ hat die Praxisleitlinie für den Umgang mit Biomarkern bei der Entscheidung \\ für die adjuvante systemische Therapie von frühen Mammakarzinomen \\ aktualisiert und präzisiert.
}

$\mathrm{H}^{2}$ auptanlass der Aktualisierung der erst im Frühjahr vergangenen Jahres frisch herausgegebenen Leitlinien zum Einsatz von Biomarkern in der adjuvanten Behandlung von Mammakarzinompatientinnen sind die Erkenntnisse aus der Studie MINDACT (Microarray In Node-negative and 1 to 3 positive lymph node Disease may Avoid ChemoTherapy) zum Nutzen des MammaPrint-Tests bei Brustkrebs im Frühstadium. Der Test analysiert die Expression von 70 Genen im Tumorgewebe, die Auswertung erfolgt zentral in einem Labor in Amsterdam. Nur in Nordamerika gibt es ein eigenes zertifiziertes Labor in Irvine, Kalifornien. Im Ergebnis der Genanalyse wird den betroffenen Patientinnen ein niedriges oder hohes Risiko zugewiesen, in den kommenden fünf beziehungsweise zehn Jahren Fernmetastasen zu entwickeln.

An MINDACT waren knapp 6.700 Patientinnen beteiligt, die an invasivem Brustkrebs im Stadium T1, T2 oder im operablen Stadium T3 erkrankt waren und nicht mehr als drei positive Lymphknoten aufwiesen. Das genomische Risiko der Frauen wurde mittels MammaPrint geschätzt. Darüber hinaus erfolgte eine klinisch-pathologische Abschätzung des Risikos nach gängigen Kriterien für und wider eine adjuvante Chemotherapie, wie Rezeptorstatus, Differenzierungsgrad und Lymphknotenbefall.

Interessant waren vor allem die Fälle mit einer divergierenden Risikoaussage (23,2\% der Frauen). Diese Patientinnen wurden nach dem Zufallsprinzip entweder wegen des hohen klinischen beziehungsweise genomischen Risikos chemotherapiert oder wegen des geringen genomischen beziehungsweise klinischen Risikos nicht adjuvant systemisch behandelt. Von den Frauen mit hohem klinischem und niedrigem genomischem Risiko, die aus klinischen Gründen eine Chemotherapie erhielten, lebten nach fünf Jahren noch 95,9\% metastasenfrei. Bei Frauen dieser Gruppe, die wegen des geringen genomischen Risikos keine Chemotherapie bekommen hatten, lag dieser Anteil bei 94,4\%. Die Differenz war statistisch nicht signifikant $(p=0,27)$. Die entsprechenden Quoten von Patientinnen mit niedrigem klinischem Risiko und hohem genomischem mit und ohne Chemotherapie lagen ebenfalls bei 95,8\% bzw. 95,0\%.

Bei niedrigem klinischem Risiko bietet der Test des Genoms offenbar keinen Vorteil für die Therapieentscheidung. Vielmehr sind es die Frauen mit klinisch hohem Risiko, die von MammaPrint profitieren, da das Ergebnis hier zwischen pro und contra adjuvante Chemotherapie entscheiden hilft.

Aus den MINDACT-Resultaten leiten die ASCO-Experten folgende Empfehlungen ab:

_Bei Patientinnen mit Hormonrezeptor-positivem, HER2-negativem und Lymphknoten-negativem Brustkrebs, die ein hohes klinisches Risiko aufweisen, kann MammaPrint helfen, jene Patientinnen mit guter Prognose zu identifizieren, die potenziell nur einen begrenzten Nutzen aus einer Chemotherapie ziehen.

- Patientinnen mit Hormonrezeptorpositivem, HER2-negativem und Lymphknoten-negativem Brustkrebs, die ein geringes klinisches Risiko aufweisen, sollten nicht mit MammaPrint getestet werden, weil ihre Prognose ohnehin gut ist und durch Chemotherapie nicht verbessert wird, selbst wenn das genomische Risiko hoch ist.

_ Bei Patientinnen mit Hormonrezeptor-positivem, HER2-negativem
Brustkrebs und ein bis drei positiven Lymphknoten, die ein hohes klinisches Risiko aufweisen, kann MammaPrint helfen, jene Patientinnen mit einer guten Prognose zu identifizieren, die potenziell nur einen begrenzten Nutzen aus einer Chemotherapie ziehen. Allerdings sollte den Frauen deutlich gesagt werden, dass ein Nutzen der systemischen Therapie nicht auszuschließen ist, vor allem dann, wenn mehr als ein Lymphknoten befallen ist.

- Bei Patientinnen mit Hormonrezeptor-positivem, HER2-negativem Brustkrebs und ein bis drei positiven Lymphknoten, die ein niedriges klinisches Risiko aufweisen, sollte MammaPrint nicht eingesetzt werden. Die Daten zum Nutzen reichen hier nicht aus.

_ Bei Patientinnen mit HER2-positivem Brustkrebs sollte MammaPrint nicht eingesetzt werden. Hier sind zunächst weitere Studien nötig.

- Bei Patientinnen mit triple-negativem Brustkrebs sollte MammaPrint nicht eingesetzt werden, um über die adjuvante Chemotherapie zu entscheiden.

Am Ende fügte das Gremium seinen Ausführungen allerdings noch ein Cave an. Es gebe verschiedene Genassays, die für die Entscheidung, auf eine adjuvante Chemotherapie zu verzichten, nützlich seien, schreiben die ASCO-Fachleute. Doch keiner der Tests sei vollkommen. Manche Patientinnen hätten trotz günstiger Testergebnisse Rückfälle erlitten. Und viele Patientinnen mit einer gemäß Genanalyse düsteren Prognose blieben selbst dann krankheitsfrei, wenn sie nicht chemotherapiert würden. „Wir benötigen deshalb weitere Fortschritte bei den Testverfahren, um einerseits Übertherapie zu reduzieren und andererseits das Rezidivrisiko zu minimieren“, schlussfolgert das Expertengremium.

Dr. Robert Bublak

Krop I et al. Use of Biomarkers to Guide Decisions on Adjuvant Systemic Therapy for Women With Early-Stage Invasive Breast Cancer:

American Society of Clinical Oncology Clinical

Practice Guideline Focused Update.

J Clin Oncol. 2017;24:2838-47 